cef

\section{moderate} variation of cell length and cell age seems to combine to contribute to rifampicin tolerance פ

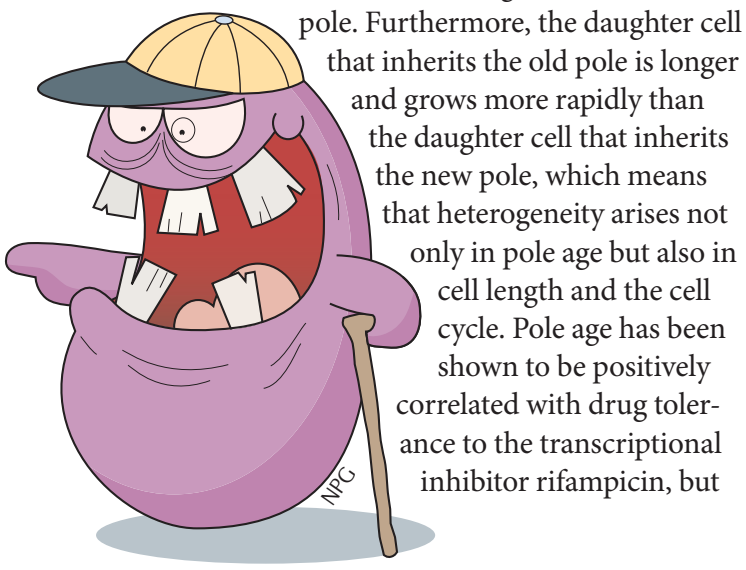

Clonal populations of Mycobacterium tuberculosis can exhibit differential responses to drug treatment, owing to phenotypic heterogeneity that produces drug-susceptible and drugtolerant subpopulations. However, the factors that underlie the heterogeneity of drug responses in M. tuberculosis are not well understood. Using single-cell imaging to study phenotypically heterogeneous populations, Richardson et al. now show that cell length and cell age are important factors associated with mycobacterial tolerance to rifampicin.

The rod-shaped cells of Mycobacterium spp. grow and divide asymmetrically, which has important consequences for phenotypic heterogeneity. When a cell divides, daughter cells inherit one pole from the parent cell and create one new distal pole. Growth occurs at both poles, but elongation at the old pole is faster than elongation at the new pele. Furthermore, the daughter cell and grows more rapidly than the daughter cell that inherits the new pole, which means that heterogeneity arises not pole age but also in cycle. Pole age has been no to bitively correlated with drug tolernce to the transcriptiona inhibitor rifampicin, but

BACTERIAL PATHOGENESIS

\title{
Mycobacteria that are long in the tooth
}

it is not known whether this correlation is directly due to the age or growth rate of the pole, or instead due to other cellular characteristics that may be confounding factors.

Richardson et al. used single-cell imaging to capture heterogeneity in a rifampicin-treated population of Mycobacterium smegmatis, which is a faster-growing, non-pathogenic laboratory model of $M$. tuberculosis. The authors found that the population could not only be divided into drugtolerant and drug-susceptible cells, but that the drug-susceptible cells could further be divided into 'dead' cells and 'zombie' cells. Both 'dead' cells and 'zombie' cells seemed to have lost viability, as suggested by disruption to their membranes and a failure to recover growth, but the two subpopulations could be distinguished by the ability of 'zombie' cells to complete cell division before the cessation of growth, whereas the cessation of growth in 'dead' cells was immediate.

A fluorescent reporter of single-stranded DNA enabled the simultaneous study of several cellular characteristics. Using datadriven modelling to separate the contributions of individual factors to the overall phenotype, the authors found that drug tolerance directly correlated with a long cell length at birth, which was responsible for an indirect correlation with pole age. Thus, cell length, rather than pole age, seems to be an important contributor to rifampicin tolerance. Interestingly, 'dead' and 'zombie' cells exhibited strikingly different cellular characteristics: 'dead' cells were shorter $(3.9 \pm 1.2 \mu \mathrm{m})$ and younger $(0.9 \pm 1.0 \mathrm{~h})$ than tolerant cells $(5.3 \pm 1.1 \mu \mathrm{m} ; 1.5 \pm 1.1 \mathrm{~h})$, whereas 'zombie' cells were longer $(5.6 \pm 1.6 \mu \mathrm{m})$ and older $(2.4 \pm 1.0 \mathrm{~h})$. Despite these differences, both 'dead' and 'zombie' cells were susceptible to drug treatment.

Together, the findings of the study suggest that mycobacterial tolerance to rifampicin is not directly associated with pole age. Instead, moderate variation of cell length and cell age seems to combine to contribute to rifampicin tolerance. However, the authors note that these two factors could only predict $60 \%$ of the heterogeneity of drug response, which, although a substantial improvement on the $37 \%$ accuracy expected by chance, suggests that additional contributing factors await discovery. Thus, mycobacterial tolerance to rifampicin is probably associated with a complex set of variables that underlie the overall physiology of the cell.

Naomi Attar

ORIGINAL ARTICLE Richardson, K. et al. Temporal and intrinsic factors of rifampicin tolerance in mycobacteria. Proc. Natl Acad. Sci. USA http://www.pnas.org/cgi/doi/10.1073/ pnas.1600372113 (2016) 